\title{
Nanoparticles in magnetic resonance imaging: from simple to dual contrast agents
}

REVIEW

\author{
This article was published in the following Dove Press journal: \\ International Journal of Nanomedicine \\ 6 March 2015 \\ Number of times this article has been viewed
}

\author{
Joan Estelrich ${ }^{1,2}$ \\ María Jesús Sánchez-Martín' \\ Maria Antònia Busquets ${ }^{1,2}$ \\ 'Departament de Fisicoquímica, \\ Facultat de Farmàcia, Universitat \\ de Barcelona, Barcelona, Catalonia, \\ Spain; ${ }^{2}$ Institut de Nanociència I \\ Nanotecnologia (IN²UB), Barcelona, \\ Catalonia, Spain
}

\begin{abstract}
Magnetic resonance imaging (MRI) has become one of the most widely used and powerful tools for noninvasive clinical diagnosis owing to its high degree of soft tissue contrast, spatial resolution, and depth of penetration. MRI signal intensity is related to the relaxation times ( $T_{1}$, spin-lattice relaxation and $T_{2}$, spin-spin relaxation) of in vivo water protons. To increase contrast, various inorganic nanoparticles and complexes (the so-called contrast agents) are administered prior to the scanning. Shortening $T_{1}$ and $T_{2}$ increases the corresponding relaxation rates, $1 / T_{1}$ and $1 / T_{2}$, producing hyperintense and hypointense signals respectively in shorter times. Moreover, the signal-to-noise ratio can be improved with the acquisition of a large number of measurements. The contrast agents used are generally based on either iron oxide nanoparticles or ferrites, providing negative contrast in $T_{2}$-weighted images; or complexes of lanthanide metals (mostly containing gadolinium ions), providing positive contrast in $T_{1}$-weighted images. Recently, lanthanide complexes have been immobilized in nanostructured materials in order to develop a new class of contrast agents with functions including blood-pool and organ (or tumor) targeting. Meanwhile, to overcome the limitations of individual imaging modalities, multimodal imaging techniques have been developed. An important challenge is to design allin-one contrast agents that can be detected by multimodal techniques. Magnetoliposomes are efficient multimodal contrast agents. They can simultaneously bear both kinds of contrast and can, furthermore, incorporate targeting ligands and chains of polyethylene glycol to enhance the accumulation of nanoparticles at the site of interest and the bioavailability, respectively. Here, we review the most important characteristics of the nanoparticles or complexes used as MRI contrast agents.
\end{abstract}

Keywords: gadolinium, iron oxide nanoparticles, magnetoliposomes, paramagnetic nanoparticles, superparamagnetic nanoparticles, relaxivity

\section{Introduction}

Imaging is widely used in scientific and technological applications because of the interface it provides between vision and intuition. In particular, biological imaging is a rapidly growing field, not only in fundamental biology but also in medical science. ${ }^{1}$ Recently, biomedical imaging has received enormous attention in view of its capacity to aid analysis and diagnosis through images at the molecular and cellular levels. ${ }^{2}$ As a result, a new discipline, known as "molecular imaging" (MI) has emerged, which combines molecular biology and in vivo imaging. ${ }^{3}$ The aim of $\mathrm{MI}$ is to monitor and measure biological processes in living subjects via spectral data. The measurement and monitoring of biological processes provides information similar to that from a biopsy, but it is noninvasive and performed in real time, thereby offering the possibility of sequential and longitudinal monitoring. The use of MI techniques permits molecular changes associated with the onset and development of pathologic states to be quantified, and the approach can provide early diagnosis and prognosis of diseases
Correspondence: Maria Antònia Busquets Departament de Fisicoquímica, Facultat de Farmàcia, Universitat de Barcelona, Avda Joan XXIII s/n, 08028-Barcelona, Catalonia, Spain

Tel +34934024556

Fax +34934035987

Email mabusquetsvinas@ub.edu
International Journal of Nanomedicine 20 I5:I0 I727-I74I

1727

Dovepress

http://dx.doi.org/10.2147/IJN.S76501 (c) (i) () 2015 Estelrich et al. This work is published by Dove Medical Press Limited, and licensed under Creative Commons Attribution - Non Commercial (unported, v3.0) License. The full terms of the license are available at http://creativecommons.org/licenses/by-nd/3.0/. Non-commercial uses of the work are permitted without any further Medical Press Limited, provided the work is properly attributed. Permissions beyond the scope of the License are administered by Dove Medical Press Limited. Information on how to request permission may be found at: http://www.dovepress.com/permissions.php 
such as cancer. Other applications include the evaluation of the response to therapy, and the study of biological processes in living subjects. ${ }^{4-6}$ Traditional MI modalities include X-ray computed tomography (CT), optical imaging (OI), positron emission tomography (PET), single-photon emission CT (SPECT), ultrasound, and magnetic resonance imaging (MRI). ${ }^{7,8}$ Several promising new imaging modalities, such as fluorescence-mediated tomography and photoacoustic tomography, are currently under development. ${ }^{9}$ Each of these diagnostic modalities has its advantages and disadvantages. For example, MRI and CT have high spatial resolution and are able to provide detailed anatomical information, but they lack sensitivity. In contrast, PET and SPECT are highly sensitive, but have limited resolution and cannot provide anatomical information.

Through the development of highly specialized and efficient contrast agents, MRI has evolved into a versatile technique with multiple functions and has become one of the most powerful noninvasive imaging tools in the biomedical toolbox. High resolution and excellent soft tissue contrast are its main advantages over other in vivo imaging techniques. MRI relies on large magnetic fields and radio frequencies (RFs), and makes use of the relaxation times of protons in mobile molecules such as water, lipids, and proteins that are present in organs at different concentrations, to produce high-resolution soft tissue anatomical images with good endogenous contrast. ${ }^{10}$ In the following sections, we review many of the most innovative approaches that have been adopted in the recent history of MRI contrast agents based on nanoparticles; mainly on superparamagnetic iron oxide nanoparticles. In this mini-review, we also include polynuclear or particulate contrast agents that are the result of progression from previous ionic agents. This is the case of the chelates of gadolinium and manganese oxide that were developed from experience with previous $\mathrm{Gd}^{3+}$ - and $\mathrm{Mn}^{2+}$-based agents respectively. In contrast, we have excluded from this review diamagnetic chemical exchange saturation transfer (CEST) and paramagnetic CEST (PARACEST) agents, although they can be included in nanoparticulate systems such as liposomes and polymers. ${ }^{11-13}$

The use of nanoparticles as imaging probes has several advantages over conventional imaging agents. Loadability is one of the advantages where the concentration of the imaging agent can be controlled within each nanoparticle during the synthesis process. Another advantage is the tunability of the surface of the nanoparticles that can potentially extend the circulation time of the agent in the blood or target a specific location within the body. Finally, nanoparticles can act as multifunctional MI agents, since they have two or more properties that can be used simultaneously in multiple imaging techniques, and especially in MRI. ${ }^{14}$

\section{Magnetic resonance imaging: origin of the contrast}

When a strong magnetic field is applied to a sample (in clinical diagnosis, magnetic fields of 1.5 or $3 \mathrm{~T}$ are usually used), the magnetic field aligns the magnetic moments of protons in the sample, producing an equilibrium magnetization along the longitudinal axis. A RF pulse, at a resonant frequency $(5-100 \mathrm{MHz})$ capable of transferring energy to protons, can then rotate their magnetic moments away from the longitudinal axis, in phase, to an angle called the flip angle. Upon removal of the radiation, the magnetic moments of the protons relax to equilibrium. ${ }^{15}$ In MRI, this process is repeated in a quick succession of RF pulses. The time taken by the magnetic moments to return to their original alignment with the magnetic field is called the relaxation time, and it is tissue dependent. This relaxation can be divided into two different, independent processes: 1) longitudinal relaxation (characterized by the parameter $T_{1}$ ) and 2) transverse relaxation (characterized by the parameter $T_{2}$ ).

$T_{1}$, called the spin-lattice relaxation time, relates to how fast the magnetization parallel to the static magnetic field recovers after a perturbation is applied to the system. Protons that relax rapidly (short $T_{1}$ ) recover full magnetization along the longitudinal axis quickly and produce high signal intensities. For protons that relax more slowly (long $T_{1}$ ), full magnetization along the longitudinal axis is not recovered before subsequent RF pulses, and so they inherently produce a lower intensity signal. ${ }^{15}$

$T_{2}$ relates to how rapidly the magnetization in the plane perpendicular to the static magnetic field loses coherence. During an RF pulse, proton nuclei spin in phase with each other, whereas after the pulse, the magnetic fields of all the nuclei interact with each other, and energy is exchanged between them. As a consequence, the nuclei lose their phase coherence and tend to spin in a random fashion. ${ }^{16}$ Because $T_{2}$ decay is the result of the exchange of energy between spinning protons, it is referred to as spin-spin relaxation.

Longitudinal and transverse relaxation processes are executed independently and simultaneously, although $T_{2}$ is usually much shorter than $T_{1}$, and this difference allows tissues to be differentiated. ${ }^{17}$ In most cases, the combination of the intrinsic molecular interactions of neighboring molecules and extrinsic magnetic field inhomogeneities means that the observed transverse relaxation time $\left(T_{2}^{*}\right)$ 
is even shorter than the natural $T_{2}$ that would be caused by pure spin-spin interactions. To eliminate external magnetic field effects and generate the real $T_{2}$-weighted images based purely on molecular interactions, a spin-echo sequence is used. This uses $90^{\circ}$ pulses to excite the magnetization and one or more $180^{\circ}$ pulses to refocus the spins and generate signal echoes named spin echoes. ${ }^{15}$

The endogenous MRI contrast in soft tissue comes from local differences in the proton density (water concentration) resulting in different values of $T_{1}$ and $T_{2}$. On this basis, endogenous contrast depends on the chemical and physical nature of the tissues. ${ }^{18}$ Despite the relatively high quality of such images of soft tissues, in some cases there is not enough image contrast to diagnose the pathology of interest. In these circumstances, the low endogenous sensitivity can be enhanced by increasing the magnetic field (from 3 to $7 \mathrm{~T}$ and beyond), acquiring data for longer or designing more sensitive sequences and probes. An important alternative is to use exogenous contrast agents. ${ }^{10}$

\section{Exogenous contrast agents}

Contrast agents have a wide variety of chemical compositions. They can be small mononuclear or polynuclear paramagnetic chelates; metalloporphyrins; polymeric or macromolecular carriers of covalently or noncovalently bonded paramagnetic chelates; particulate contrast agents (including fluorinated or nonfluorinated paramagnetic micelles or liposomes) and paramagnetic or superparamagnetic particles (eg, iron oxides and $\mathrm{Gd}^{3+}$-labeled zeolites); diamagnetic CEST polymers; diamagnetic hyperpolarization probes (gases and aerosols); and ${ }^{13} \mathrm{C}$-labeled compounds or ions (eg, $\left.6 \mathrm{Li}^{+}\right) .{ }^{19}$ The main role of $T_{1}$ and $T_{2}$ contrast agents in MRI is to shorten selectively the relaxation times of water protons in the region of interest and thus to provide better contrast for anatomical regions. Contrast is enhanced when one tissue has either higher affinity for the contrast agents or higher vascularity than another. Diseased tissues, such as tumors, are metabolically different from healthy tissues and have taken up the contrast agent in different ways, resulting in a contrast in MRI images. ${ }^{19,20} T_{1}$-weighted images illustrate anatomy well and are preferred when a clear image of the structures is required. $T_{2}$-weighted images produce good pathological information since collections of abnormal fluid appear brighter than the normal tissue background.

Although nearly all MRI contrast agents affect both $T_{1}$ and $T_{2}$, the effects of contrast agents are usually more pronounced for either $T_{1}$ or $T_{2}$, leading to their categorization as either $T_{1}$ or $T_{2}$ contrast agents. Contrast enhancement is

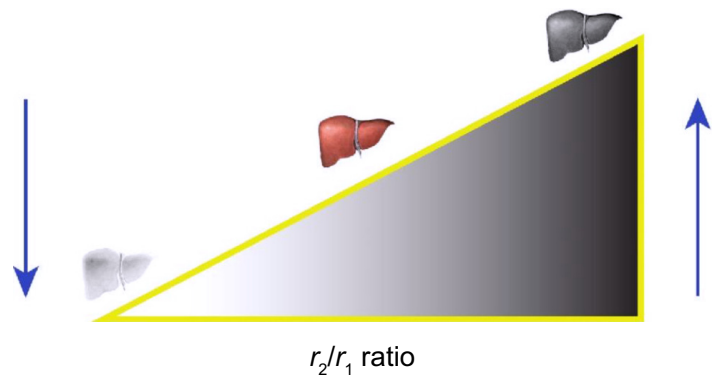

Figure I Influence of the $r_{2} / r_{1}$, ratio on the efficiency of a contrast agent. Notes: High values of $r_{2} / r_{1}$ are characteristic of $T_{2}$ contrast agents, which produce a hypointense signal in $T_{2}$-weighted images, and thus organs appear darker in the image. Low values of $r_{2} / r_{1}$ define $T_{1}$ contrast agents, and the associated images are clearer and brighter.

measured by the relaxation rate $R_{i}=1 / T_{i}\left(\mathrm{~s}^{-1}\right)$, where $i=1$ or 2 . The most important parameter for defining the efficiency of a contrast agent is its relaxivity, $r_{i}\left(r_{i}=R_{i} / c_{\mathrm{CA}}\left(\mathrm{mM}^{-1} \cdot \mathrm{s}^{-1}\right)\right.$, where $c_{\mathrm{CA}}$ is the analytical concentration of ion responsible for the contrast). The $r_{2} / r_{1}$ ratio is also used to indicate the contrast efficiency; the higher the ratio, the greater the efficiency of a $T_{2}$ contrast agent and vice versa for a $T_{1}$ contrast agent (Figure 1).

The relaxivity for an MRI contrast agent is defined as the increase in the relaxation rate of the solvent (water) induced by $1 \mathrm{mmol} \cdot \mathrm{L}^{-1}$ of the active ion of the contrast agent, and it is calculated according to

$$
r_{i, \mathrm{obs}}=\left[\frac{1}{T_{i, \mathrm{obs}}}-\frac{1}{T_{i, \text { water }}}\right] / c_{\mathrm{CA}}
$$

The relaxivity is dependent on the magnetic field applied and the temperature, so it should be reported together with both these parameters.

Most $T_{1}$ contrast agents currently available are paramagnetic complexes, while those classed as $T_{2}$ contrast agents are mostly superparamagnetic iron oxides. ${ }^{18}$

\section{$T_{\text {, contrast agents }}$}

The first generation of exogenous $T_{1}$ contrast agents (also called positive contrast agents) consisted of high-spin paramagnetic metal ions such as manganese $\left(\mathrm{Mn}^{2+}\right)$, iron $\left(\mathrm{Fe}^{3+}\right)$, or gadolinium $\left(\mathrm{Gd}^{3+}\right){ }^{21}$ These contrast agents produce hyperintense signals in $T_{1}$-weighted images. To obtain significant changes in proton relaxation and therefore a good contrast, the paramagnetic ion needs to be in close contact with the protons of the surrounding water molecules. However, owing to the toxicity associated with these cations (eg, transient destruction of professional macrophages, exchange with endogenous calcium ions, etc), they are used 
after complexation with low-molecular-weight chelating molecules with no explicit core and surface coating.

$\mathrm{Gd}$ is the most clinically used metal ion in paramagnetic $T_{1}$ contrast agents. It possesses an electron spin of $7 / 2$ and hence seven unpaired electrons promoting spin relaxation due to flipping spins and rotational motion. Free Gd ions are cytotoxic and are retained in liver, spleen, and bone. ${ }^{22}$ To avoid this toxicity, a chelating process is applied to $\mathrm{Gd}$, in which large organic molecules form a stable complex around the Gd. The chelate reduces the chances of toxicity that result from exposure to Gd. The stable complex is predominantly eliminated via the kidneys. Examples of chelating compounds are diethylene-triamine-pentaacetic acid (DTPA), 1,4,7,10tetraazacyclo-dodecane-1,4,7,10-tetraacetic acid (DOTA), and dipyridoxyl-di-phosphate (DPDP). ${ }^{19}$ The chemical structures of such $T_{1}$ agents are typically characterized by neutral or anionic metal complexes of the type $\left[\mathrm{M}\left(\mathrm{H}_{2} \mathrm{O}\right)(\mathrm{L})\right]$ or $\left[\mathrm{M}\left(\mathrm{H}_{2} \mathrm{O}\right)\right.$ $(\mathrm{L})]^{n-}$, where $\mathrm{M}$ is the paramagnetic $\mathrm{Gd}^{3+}$ or $\mathrm{Mn}^{2+/ 3+}$ ion, and $\mathrm{L}$ a macrocyclic or acyclic polyaminopolycarboxylate. ${ }^{23}$

Different types of Gd-containing contrast agents have been approved by the European Medicines Agency (EMEA) and the US Food and Drug Administration (FDA) (Table 1) for use in MRI as a contrast agent to provide improved images of organs and tissues.

However, although Gd is regarded as safe when administered as a chelated compound, the use of some Gd chelates in people with renal disease has been linked to a rare but severe complication; the medical condition referred to as "nephrogenic systemic fibrosis." For this reason, the World Health Organization (WHO) issued a restriction on the use of Gd contrast agents, informing that these compounds were contraindicated in patients with chronic severe renal insufficiency, in those with acute renal insufficiency of any severity due to hepatorenal syndrome, or in the perioperative liver transplantation period, and in newborn babies up to 4 weeks of age. ${ }^{24}$

Because of their low molecular weight, conventional Gd-based contrast agents are mostly nonspecific extracellular contrast agents and exhibit rapid extravasation from the vascular space. In this way, after being intravenously injected, these agents rapidly leak from the blood pool into the interstitium with a distribution half-life $\left(t_{1 / 2}\right)$ of about $5 \mathrm{~min}$. They are mainly cleared by the kidneys with an elimination $t_{1 / 2}$ of about $80 \mathrm{~min} .{ }^{19}$ This limitation, which is inherent to MRI, is known as the partial volume dilution effect, and involves a loss of apparent activity in small objects or regions because of the limited resolution of the imaging system. The partial volume dilution effect has often led to the failure of targeted contrast in vivo. ${ }^{25}$ Extracellular agents are typically Gd chelates of linear or macrocyclic polyaminocarboxylate ligands, and constitute the most important class of MRI contrast agents available. Initial attempts to target MRI focused on coupling Gd atoms directly to antibodies or proteins, but these approaches delivered insufficient paramagnetic material to effectively decrease local relaxation times, and provided inadequate MR signal enhancement in $T_{1}$ images at typical clinical field strengths. Moreover, for certain purposes such as MR angiography (MRA: a special type of MRI used to study blood vessels) the time window for contrast-enhanced images is very narrow, due to rapid extravasation, which limits the acquisition of high-resolution images. For MRA, contrast agents must be blood-pool agents (also known as intravascular contrast agents) and are characterized by their high molecular weight $(<20 \mathrm{kDa})$ and higher relaxivities. Their large size prevents diffusion through the vascular

Table I Gadolinium-based contrast agents approved for use in humans by the EMEA or FDA

\begin{tabular}{lllll}
\hline Generic name & Chemical or code name & Type of agent & Product name & Health agency of approbation \\
\hline $\begin{array}{l}\text { Gadofosveset trisodium } \\
\text { Gadoxetate disodium }\end{array}$ & MS-325 & Blood pool & Ablavar & FDA/EMEA (I) \\
& Gd-EOB-DTPA & Targeting & $\begin{array}{l}\text { Eovist (formerly Vasevist) } \\
\text { FDA }\end{array}$ \\
Gadopentetate dimeglumine & Gd(DTPA) & Nonspecific extracellular & Magnevist & FMEA (2) \\
& & & Magnegita & EMEA (I) \\
Gadodiamide & Gd-DTPA-BMA & Nonspecific extracellular & Omniscan & EMEA (I) \\
Gadoversetamide & Gd-DTPA-BMEA & Nonspecific extracellular & OptiMark & FDA/EMEA (I) \\
Gadoteridol & Gd-HP-DO3A & Nonspecific extracellular & ProHance & FDA/EMEA (3) \\
Gadobenate disodium & Gd-BOPTA & Targeting & MultiHance & FDA/EMEA (2) \\
Gadoterate & Gd-DOTA & Nonspecific extracellular & Dotarem & FDA/EMEA (3) \\
Gadobutrol & Gd-DO3A-butrol & Nonspecific extracellular & Gadovist & FDA/EMEA (3) \\
\hline
\end{tabular}

Notes: EMEA classification of the contrast agents in relation to the risk of nephrogenic systemic fibrosis: (1) high risk; (2) medium risk; (3) low risk. Abbreviations: EMEA, European Medicines Agency; FDA, US Food and Drug Administration; BMA, bis-methylamide; BMEA, bis-methoxiethylamide; BOPTA, benzyloxypropionictetracetate; DOTA, I,4,7,10-tetraazacyclo-dodecane-I,4,7,Io tetracetic acid; EOB, ethoxybenzyl; DTPA, diethylene-triamine-pentacetic acid; Gd, gadolinium; HP-Do3A, I,4,7-tris(carboxymethyl)-10-(2'-hydroxypropyl)-I,4,7, I0-tetraazacyclododecane. 
epithelium and leakage into the interstitial space, and so they reside in the vascular system for an extended period of time. Thus, they are eliminated much more slowly from circulation than their extracellular counterparts, providing a larger imaging time window. Examples of blood-pool contrast agents are Gd-based complexes that interact noncovalently with human serum albumin, and Gd chelates complexed to polymers (eg, dextrans, polylysine derivatives, and polyamidoamines [PANAM ${ }^{\mathrm{TM}}$, GE Healthcare Institute, Waukesha, WI, USA]). ${ }^{26}$

To produce targeting agents, macromolecular constructs, such as liposomes, micelles, fluorinated nanoparticles, dendrimers, and polymers, have been prepared..$^{17,25,27}$ The resulting nanoparticles have greater paramagnetic metal surface payloads that rotate or tumble more slowly than small-molecule organometallic compounds typically used as blood-pool agents. ${ }^{28}$

$\mathrm{Mn}^{2+}$, with five unpaired electrons, is another cation used as a contrast agent. Mn-based paramagnetic nanoparticles can be classified into two categories: small-molecule agents and nanoparticulate agents. Small-molecule agents are formed by complexing Mn ions with chelates such as DPDP, DTPA, or even porphyrin rings, just as Gd chelates are. The FDA approved, in May 1997, a Mn-based contrast agent, the injectable mangafodipir trisodium $\left(\right.$ Teslacan $^{\circledR}$, St Louis MO, USA) to image the liver. However, in 2012, the EMEA was notified by the marketing authorization holder responsible for Telescan the decision to voluntarily withdrawl the marketing authorization in the European Union. ${ }^{29}$

Mn chelates can be further modified by their incorporation into lipid bilayers. Such nanoparticulate systems are made of manganese oxides such as $\mathrm{MnO}, \mathrm{MnO}_{2}$, and $\mathrm{Mn}_{3} \mathrm{O}_{4}$. After dissolution in cells due to proteolytic degradation, these particles convert from $T_{2}$ contrast agents to $T_{1}$ contrast agents. ${ }^{30}$ Although $\mathrm{Mn}^{2+}$ is a natural cellular constituent that resembles $\mathrm{Ca}^{2+}$, its toxicity is also known from dust containing $\mathrm{Mn}$ at high doses. Moreover, in view of the capacity of $\mathrm{Mn}^{2+}$ to enter cells through calcium channels, Mn complexes, dendritic Mn chelates, and even Mn nanoparticulate systems have potential applications in neuroimaging. However, this also implies that the brain may be vulnerable to Mn exposure.

One of the limitations of the majority of the contrast agents used, but that especially affects paramagnetic chelates, is that their efficiency decreases at higher magnetic fields. For example, Gd complexes are optimal at fields below $1 \mathrm{~T}$; even at the clinical field of $3 \mathrm{~T}$, the $\mathrm{T}_{1}$ relaxivity of Gd-based contrast agents is reduced by as much as one-third compared with its maximum, while at higher magnetic fields, $r_{1}$ falls to zero. ${ }^{31}$ Moreover, for in vitro cell labeling experiments or long-term in vivo cell tracking studies, the clearance of the particles needs to be far slower, which impedes the use of Gd-based chelate agents for these purposes. Hence, owing to their short blood circulation times, poor detection sensitivity, and toxicity concerns, MRI research has shifted to $T_{2}$ contrast agents, especially to superparamagnetic iron oxide nanoparticles.

\section{$T_{2}$ contrast agents}

$T_{2}$ contrast agents (or negative contrast agents) decrease the MR signal intensity of the regions they are delivered to. Consequently, they produce hypointense signals in $T_{2}$ - and $T_{2}{ }^{*}$-weighted images, ${ }^{32}$ and thus the affected regions appear darker. The phenomenon can be said to result from the large heterogeneity of the magnetic field around the nanoparticle through which water molecules diffuse, since diffusion induces dephasing of the proton magnetic moments, resulting in $T_{2}$ shortening. $T_{2}$ contrast agents are also called susceptibility agents because of their effect on the magnetic field. $T_{2}$ shortening is a remote effect, whereas the $T_{1}$ shortening process requires a close interaction between the water molecules and $T_{1}$ agents, as mentioned. ${ }^{16}$ Another difference with $T_{1}$ contrast agents is that under high magnetic fields, $R_{2}$, the relaxation rate, tends asymptotically to a positive constant. ${ }^{10}$

Iron oxide nanoparticles have been used as $T_{2}$ contrast agents for more than 25 years. These iron oxides can be ferromagnetic or superparamagnetic, depending on the size of the core of the nanoparticle. Two iron oxides are generally considered for biomedical applications: magnetite $\left(\mathrm{Fe}_{3} \mathrm{O}_{4}\right)$ and its oxidized and more stable form of maghemite $\left(\gamma-\mathrm{Fe}_{2} \mathrm{O}_{3}\right)$. The critical upper size limit for the observation of superparamagnetism is approximately $25 \mathrm{~nm}$ for magnetite and $30 \mathrm{~nm}$ for maghemite. ${ }^{33}$ The two compounds fulfill the prerequisites of: 1) chemical stability under physiological conditions, 2) low toxicity, and 3) sufficiently high magnetic moments. ${ }^{34}$

Since these two iron oxides exhibit superparamagnetic behavior, the loss of their net magnetization in the absence of an external magnetic field limits their tendency for self-aggregation, and this helps to obtain a good biological response. ${ }^{10}$ Unfortunately, the ubiquitous Van der Waals forces induce natural aggregation of the particles, and to circumvent this problem, a large portfolio of chemical approaches exists that stabilize the particles. These approaches include the modification of the surface of the particles with diverse materials. Polymers are the most 
widely used stabilizing materials, and can be classified as hydrophilic or amphiphilic, neutral or charged, homopolymers or copolymers. The polymers can be adsorbed into or anchored onto the iron oxide surface via hydrogen bonds, electrostatic forces, or pseudo-covalent bonding. Among the materials used are poly(ethylene glycol) (PEG), dextran and its derivatives, alginate, chitosan, starch, polyvinyl alcohol, albumin, poly(ethylene imine), organic siloxane, sulphonated styrene-divinyl-benzene, and bioactive molecules and structures such as liposomes. ${ }^{35,36}$

Since the biological distribution of the nanoparticles is directly dependent on their size, they have been classified according to the overall size of the particles as follows: 1) ultra-small superparamagnetic iron oxide nanoparticles (USPIONs) with diameter (d) less than $50 \mathrm{~nm}, 2$ ) superparamagnetic iron oxide nanoparticles (SPIONs) with size of hundreds of nanometers, and, ultimately, 3) micron-sized particles of iron oxide (MPIO) with a diameter higher than $1 \mu \mathrm{m} .{ }^{37}$ While the overall size of the first two classes allows them to be administered intravenously, the larger particles are usually administered orally, limiting their use to the exploration of the gastrointestinal track. There are also other formulations, such as monocrystalline iron oxide particles (MION) and cross-linked iron oxides (CLIO). ${ }^{19}$

Several SPION formulations for intravenous or oral administration have been approved for clinical use as MRI contrast agents by the EMEA and FDA; however, the majority of the compounds that were approved for intravenous administration have, at present, been taken off the market. ${ }^{38}$ Only the SPION for oral administration, Gastromark ${ }^{\circledR}$ (AMAG Pharmaceuticals, Waltham, MA, USA; ferumoxsil, silicone-coated SPIONs), is currently on the market for gastrointestinal bowel marking. The most widely applied coatings for FDA-approved SPIONs are dextran and carboxydextran. Table 2 compares the properties of nanoparticles coated with one or other polymer.

It is important to note that both the type of coating and its thickness affect the value of $r_{2}$, although the influence is unclear as studies report different effects. For instance, it has been reported that as the coating thickness increases, the ratio $r_{2} / r_{1}$ decreases. ${ }^{39}$ This is due to the inner hydrophobic layer excluding water, while the outer hydrophilic PEG layer allows water to diffuse within the coating zone. Increasing the PEG chain length leads to a reduction in $r_{2}$ values, although another study has shown that when water molecules are not excluded from regions close to the SPION core, $r_{2}$ relaxivity increases with increased chain length. ${ }^{40}$

The overall size of the particle governs its pharmacokinetics and biodistribution. Nanoparticles with a size $<5.5 \mathrm{~nm}$ are cleared by the kidneys. ${ }^{41,42}$ SPIONs whose overall diameter is larger than $200 \mathrm{~nm}$ are quickly taken up by phagocytic cells and accumulate in the monocyte phagocyte system (MPS), specifically in liver and spleen macrophages. When administered intravenously, approximately $80 \%$ of the dose is found in liver and 5\%-10\% in spleen, with it having a plasma half-life of less than 10 min. ${ }^{16}$ Therefore, such SPIONs decrease the liver and spleen signal within several minutes of administration. Malignant tumors or metastases have a lack of Kupffer cells, and due to the negligible uptake of nanoparticles, they produce a strong contrast between normal and abnormal tissue on $T_{2}$-weighted images. USPIONs evade MPS uptake and consequently increase their blood half-life $(>2 \mathrm{~h})$. This increased blood circulation maximizes the odds of SPIONs reaching their target tissue. SPIONs and USPIONs are metabolized into a soluble and nonsuperparamagnetic form of iron, which is incorporated into the normal iron pool (eg, ferritin, hemosiderin, hemoglobin) within a couple of days. ${ }^{19}$

As with any nanoparticle, SPIONs can invade small solid tumors and metastatic cells thanks to passive targeting through the enhanced permeation and retention (EPR) effect. The EPR effect aids nanoparticle uptake by way of leaky vasculature, which permits particles of nanometric size (more or less, with a hydrodynamic radius of less than $100 \mathrm{~nm}$ ) to cross from the vasculature into the interstitium. ${ }^{43}$ Poor lymphatic drainage then aids the entrapment of particles in solid tumors.

SPIONs are used as negative contrast for liver imaging, whereas the typical application of USPIONs is lymph node imaging. USPIONs have been tested as blood-pool agents

Table 2 Properties of iron oxide nanoparticles and relaxivity values of three nanoparticles coated with hydrophilic polymers

\begin{tabular}{lllllll}
\hline Name & Core material & Surface & Core size $(\mathbf{n m})$ & $\begin{array}{l}\text { Hydrodynamic } \\
\text { diameter }(\mathbf{n m})\end{array}$ & $\boldsymbol{r}_{\mathbf{2}}\left(\mathbf{m M}^{-1} \cdot \mathbf{s}^{-1}\right)$ & Magnetic field $(\mathbf{T})$ \\
\hline Ferumoxides (Feridex) & $\mathrm{Fe}_{3} \mathrm{O}_{4}-\gamma-\mathrm{Fe}_{2} \mathrm{O}_{3}$ & Dextran & 4.96 & -200 & 120 & 1.5 \\
Ferucarbotran (Resovist) & $\mathrm{Fe}_{3} \mathrm{O}_{4}$ & Carboxydextran & 4.2 & $>50$ & 186 & 1.5 \\
Ferumoxtran (Combidex) & $\mathrm{Fe}_{3} \mathrm{O}_{4}$ & Dextran & 5.85 & $<50$ & 65 & 1.5 \\
\hline
\end{tabular}

Note: Copyright $\odot 2009$ WILEY-VCH Verlag GmbH \& Co. KGaA, Weinheim. Bin Na H, Chan Song I, Hyeon T. Inorganic nanoparticles for MRI contrast agents. Adv Mater. $2009 ; 21: 2133-2148 . .^{55}$ 
because they are readily distributed in the intravascular extracellular space. In this way, USPIONs are used as contrast for lymphography ${ }^{44,45}$ and angiography, ${ }^{46,47}$ as a bone marrow contrast, or as a perfusion agent in brain and kidney.

Iron oxide nanoparticles require specific approaches to target cells other than macrophages. Since biochemical epitopes of interest are often present in nanomolar or picomolar concentrations, particle relaxivities of around $1,000,000 \mathrm{mM}{ }^{-1} \cdot \mathrm{s}^{-1}$ are required to achieve acceptable contrast-to-noise ratios at the typical field strength. ${ }^{48}$ In this way, contrast agents that target specific tissue can increase the sensitivity by increasing the local SPION concentration. To achieve such sensitivity in the nanomolar range, the surface of the SPIONs may be modified by active targeting strategies, such as the addition of ligands that are recognized by molecular signatures of afflicted cells. Polyethyleneimine (PEI) is one of the more used ligands. ${ }^{49}$ The same group ${ }^{50,51}$ has reported that hyaluronic acid targeted iron oxide nanoparticles are efficient probes for targeted MRI of cancer cells in vitro and xenografted tumor model in vivo. Other types of ligands have been studied for the targeting of such markers including antibodies, small peptides, lectins, aptamers, engineered proteins, and protein fragments. ${ }^{52}$ For instance, USPIONs of less than $10 \mathrm{~nm}$ in hydrodynamic diameter were tested for tumor-specific MRI targeting. In that study, the USPIONs were stabilized by 4-methylcatechol, and a cyclic arginine-glycine-aspartic acid (cRGD) peptide was coupled via the Mannich reaction ${ }^{53}$ (Figure 2).

The peptide RGD binds the $\alpha_{\mathrm{v}} \beta_{3}$-integrin, a cell adhesion molecule that is overexpressed in tumor vasculature and invasive tumor cells. ${ }^{54}$ After the administration of the RGDnanoparticles, the tumor MRI signal intensity decreased by $40 \% .^{53}$

One of the largest drawbacks in using SPIONs is related to the contrast mechanism that they generate. As mentioned, they are negative imaging agents, which produce a signaldecreasing effect. The resulting dark signal could be confused with other pathogenic conditions, and renders images of lower contrast than $T_{1}$ contrasted images. Moreover, the high susceptibility of $T_{2}$ contrast agents induces distortion of the magnetic field on neighboring normal tissues. This distortion of the background is called a susceptibility artifact or "blooming effect," and generates dark images with no background around the lesions. ${ }^{55}$ This effect prevents

A

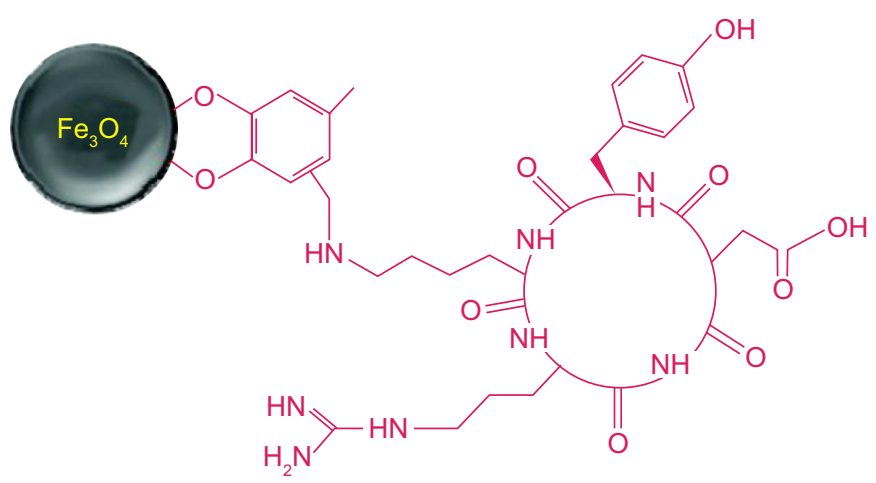

B

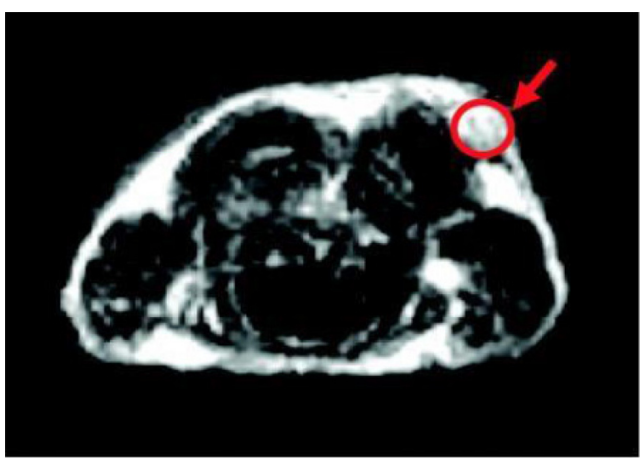

C

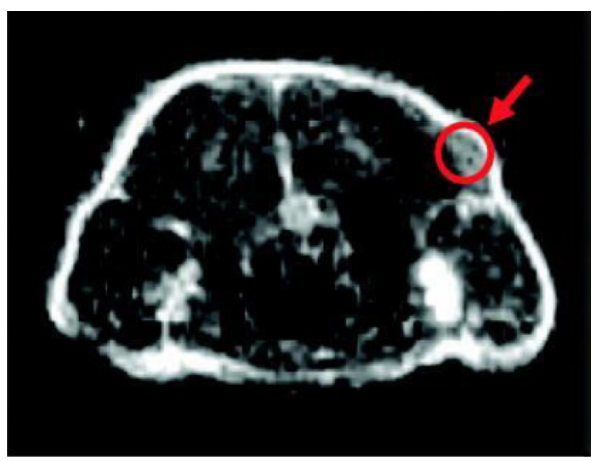

Figure 2 Schematic illustration of the coupling of cRGD peptide to the SPIONs.

Notes: (A) MRI cross-section image of the U87MG tumors implanted in mice; (B) without the nanoparticles; and (C) with the injection of $300 \mu \mathrm{g}$ of cRGD-SPIONs. Reprinted with permission from Ho D, Sun X, Sun S. Monodisperse magnetic nanoparticles for theranostic applications. Acc Chem Res. 20II;44:875-882.60 Copyright 20II American Chemical Society.

Abbreviations: CRGD, cyclic arginine-glycine-aspartic acid; SPIONs, superparamagnetic iron oxide nanoparticles; MRI, magnetic resonance imaging. 
their clinical use in low signal body regions, in organs with intrinsic high magnetic susceptibility (eg, lung), or in the presence of hemorrhagic events. ${ }^{56}$

In addition to nanoparticles whose metal core is fully based on iron oxides, other nanosystems with different magnetic cores have been introduced to improve the signal sensitivity and enhance MRI diagnostics. ${ }^{57}$ Since the transverse relaxivity $r_{2}$ depends, apart from size, on the saturation magnetization $\left(M_{\mathrm{s}}\right)$, the optimization of $M_{\mathrm{s}}$ is one of the most effective ways to achieve magnetic nanoparticles with high MRI sensitivity. It has been reported that, owing to the higher magnetization, iron nanoparticles have a higher $T_{2}$ relaxivity than analogous systems containing iron oxides. ${ }^{58}$ Alloy-based nanomaterials are good candidates for developing $T_{2}$ contrast agents with higher relaxivities. The substitution of one of the Fe ions in an iron oxide for a different magnetic atom $(\mathrm{Mn}, \mathrm{Zn}, \mathrm{Co}, \mathrm{Ni}$, etc) produces the compounds known as ferrites (Mn-ferrite, Zn-ferrite, etc) characterized by their high $M_{\mathrm{s}}$, and this enhanced $M_{\mathrm{s}}$ increases the relaxation rate. Yang et $\mathrm{al}^{59}$ have reported the suitability of Mn-ferrites as MRI contrast agents. More pronounced contrast effects are even possible when nonmagnetic ions replace the $\mathrm{Fe}$ ions. ${ }^{60}$ Interestingly, the substitution of $\mathrm{Fe}^{2+}$ with nonmagnetic $\mathrm{Zn}^{2+}$ results in an increase in the net magnetization of the nanoparticles. $M_{\mathrm{s}}$ increases with $\mathrm{Zn}^{2+}$ doping and becomes maximum with a value of $x=0.4$ in $\left(\mathrm{Zn}^{x} \mathrm{M}^{1-x}\right) \mathrm{Fe}_{2} \mathrm{O}_{4}\left(\mathrm{M}=\mathrm{Mn}^{2+}, \mathrm{Fe}^{2+}\right)$. For comparison, the $M_{\mathrm{s}}$ of $\left(\mathrm{Zn}^{0.4} \mathrm{Mn}^{0.6}\right) \mathrm{Fe}_{2} \mathrm{O}_{4}$ is $175 \mathrm{emu} \cdot \mathrm{g}^{-1}$ magnetic atom, whereas the corresponding value for $\mathrm{Fe}_{3} \mathrm{O}_{4}$ is $96 \mathrm{emu} \cdot \mathrm{g}^{-1}$ magnetic atom. The value of $r_{2}$ reaches $676 \mathrm{mM}^{-1} \cdot \mathrm{s}^{-1}$ for the $\mathrm{Zn}$-doped magnetic nanoparticles, and $98 \mathrm{mM}^{-1} \cdot \mathrm{s}^{-1}$ for $\mathrm{Fe}_{3} \mathrm{O}_{4} \cdot{ }^{61}$

Other nanoparticles with potential applications in MRI include gold-iron oxide $\left(\mathrm{Au}-\mathrm{Fe}_{3} \mathrm{O}_{4}\right)$ nanoparticles, metallic ion nanoparticles, porous hollow $\mathrm{Fe}_{3} \mathrm{O}_{4}$ nanoparticles, and Fe-based alloy nanoparticles, such as iron-cobalt (FeCo) and iron-platinum (FePt) nanoparticles. However, metallic nanoparticles are normally very reactive and are subject to fast oxidation in biological solutions. Once they are coated with a layer of polycrystalline $\mathrm{Fe}_{3} \mathrm{O}_{4}$ or a graphitic shell, these metallic nanoparticles are more stable and provide better contrast in MRI. FePt nanoparticles are chemically more stable than Fe and FeCo nanoparticles, and have been shown to have great potential as contrast agents for MRI and CT. ${ }^{28}$ However, it is worth pointing out that such systems could be used for preclinical experiments, but clinical assessment of their acute and long-term toxicity is required.

Recently, some paramagnetic ions, such as dysprosium $\left(\mathrm{Dy}^{3+}\right)$, have been proposed as good alternatives to iron oxide
$T_{2}$ contrast agents in high-field MRI, because of their high magnetic moments. ${ }^{62}$ Dy $^{3+}$ has been used as a chelate (eg, $\mathrm{Dy}^{3+}$-DTPA) or as nanoparticles (eg, $\left.\mathrm{Dy}_{2} \mathrm{O}_{3}\right) \cdot{ }^{63,64}$ One type of $\mathrm{Dy}^{3+}$-based nanoparticles are $\beta-\mathrm{NaDyF}_{4}$ nanoparticles; their relaxivity has been studied at 3 and $9.4 \mathrm{~T}$ in nanoparticles of $5.4,9.8$, and $20.3 \mathrm{~nm}$. It has been reported that their $r_{2}$ relaxivity is 6-9 times greater at $9.4 \mathrm{~T}$ than at $3 \mathrm{~T}$, and that the larger nanoparticles show higher $r_{2}$ values than the smaller ones, whereas the $r_{1}$ relaxivities are almost constant for the three sizes at 3 and $9.4 \mathrm{~T}$. At $9.4 \mathrm{~T}$, the $r_{2} / r_{1}$ ratio is 306 for nanoparticles of $20.3 \mathrm{~nm}, 230$ for those of $9.8 \mathrm{~nm}$, and 106 for those of $5.4 \mathrm{~nm}$.

One important result of classical outer-sphere relaxation theory ${ }^{65}$ is that the $r_{2} / r_{1}$ ratio increases with increasing particle size, and thus, smaller particles are much better $T_{1}$-shortening agents then larger ones (Figure 3).

As a consequence of their larger size and magnetic moments, SPIONs were initially developed as $T_{2}$-agents. However, a new generation of USPIONs, with diameters less than $10 \mathrm{~nm}$, has also been reported to have excellent $T_{1}$-enhancing properties. ${ }^{28,66}$ Compared with paramagnetic ions, SPIONs have higher molar relaxivities, and, when used as blood-pool and tissue-specific agents, may offer advantages at low concentrations. ${ }^{67}$

As indicated above, SPIONs generate dark or negative contrast at the target site with a marked blooming effect from magnetite susceptibility artifacts. Moreover, for MI applications, persistent $T_{2}^{*}$ effects from circulating SPIONs delay MRI by 24-72 h after injection.

To overcome these limitations, specific off-resonance pulse sequences capable of generating a bright contrast in the presence of SPIONs have been proposed. ${ }^{68,69}$ More recently, another approach, called inversion recovery ON-resonant water suppression (IRON)-MRI, has been developed to

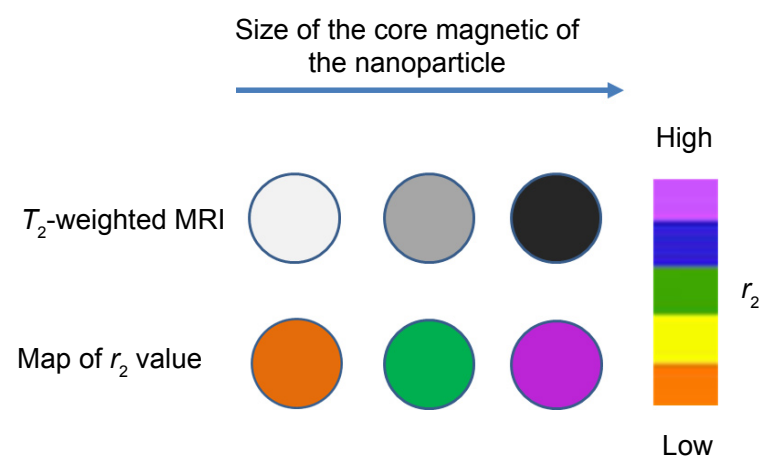

Figure $3 T_{2}$-weighted contrasts and $r_{2}$ color maps for iron oxide nanoparticles of different size. 
obtain a positive contrast. ${ }^{70}$ Unfortunately, these techniques do not eliminate the signal loss or magnetic susceptibility artifacts, but rather exploit them to generate more easily perceived bright images of the contrast effects, often at the expense of the surrounding anatomical detail. Moreover, the undesirable 24-72 h delay between injection of the agent and the imaging result remains. One approach to resolve the prolonged delay between treatment and imaging has been to consider the use of microparticles of iron oxide (MPIO, size range $0.76-1.63 \mu \mathrm{m}$ ), which rapidly pass into MPS organs. ${ }^{71}$ However, such large iron oxide particles were prone to aggregation and rapid pulmonary entrapment, and despite the leukocyte mimicking dual homing ligand approach proposed, the potential of the approach will depend on overcoming many challenges. ${ }^{72,73}$

A more recent approach ${ }^{28}$ is the preparation of a colloidal iron oxide nanoparticle platform (CION), which is achieved by embedding oleate-coated magnetite particles in a hydrophobic matrix composed of vegetal oil and partially cross-linked phospholipids. Contrary to expectations, this formulation decreased $T_{2}$ effects, thus allowing positive $T_{1}$-weighted contrast detection. A CION may be used to detect biosignatures via voxels at very low nanomolar densities.

\section{Dual $\left(T_{1}\right.$ and $\left.T_{2}\right)$ contrast agents}

Conventional MRI contrast agents are mostly effective only in a single imaging mode: either $T_{1}$ or $T_{2}$. They frequently result in ambiguities in diagnostics, especially when the biological targets are small. The combination of simultaneously strong $T_{1}$ and $T_{2}$ contrast effects in a single contrast agent could be a new breakthrough, since it can potentially provide more accurate MRI via self-confirmation with better differentiation of normal and diseased areas. Dual contrast agents would eliminate the possible ambiguity of a singlemode contrast agent when some of the in vivo artifacts are present. ${ }^{57}$ However, the production of such a contrast has proved extremely challenging. ${ }^{74}$

USPIONs with a core of less than $10 \mathrm{~nm}$ in diameter are capable of producing positive contrast in $T_{1}$-weighted images when administered in moderate concentrations. ${ }^{75,76}$ While positive $T_{1}$ contrast is possible with USPIONs, this benefit is at the expense of their $T_{2}$ effects. ${ }^{77,78}$ For this reason, mixing both types of iron oxides, SPIONs and USPIONs, to form a single contrast agent could potentially be a good choice. However, an important problem arises as a consequence of the strong magnetic coupling between the $T_{1}$ and $T_{2}$ contrast agents when they are in close proximity: the spin-lattice relaxation process of $T_{1}$ contrast materials is significantly diminished. One strategy to overcome this phenomenon is the inclusion of a separation layer to modulate the magnetic coupling. To this end, micellar structures incorporating organic block copolymers, inorganic porous materials, and core-shell-type inorganic materials have been considered as possible frameworks. ${ }^{52}$ For instance, a core-shell-type $T_{1}-T_{2}$ dual-mode nanoparticle contrast has been described, where the $T_{1}$ contrast material, $\mathrm{Gd}_{2} \mathrm{O}\left(\mathrm{CO}_{3}\right)_{2}$ of $1.5 \mathrm{~nm}$, is located on the shell so as to come into direct contact with water molecules, for high $T_{1}$ contrast effects; while the superparamagnetic $T_{2}$ contrast material, $\mathrm{MnFe}_{2} \mathrm{O}_{4}$ of $15 \mathrm{~nm}$, is located at the core, from where it induces a long-range magnetic field for the relaxation of water molecules. The two materials are separated by $\mathrm{SiO}_{2}$. By adjusting the thickness of the $\mathrm{SiO}_{2}$, the magnetic coupling between the $T_{1}$ and $T_{2}$ contrast agents is controlled. As the $\mathrm{SiO}_{2}$ becomes thicker, $T_{1}$ quenching reduces and, concurrently, $r_{1}$ increases; while the decrease in the $T_{2}$ effects is relatively weaker. When the $\mathrm{SiO}_{2}$ layer is $16 \mathrm{~nm}$ thick, both $T_{1}$ and $T_{2}$ contrast effects become larger than the effects of the individual single-mode contrast effects (Figure 4). ${ }^{79}$

In other studies, colloidal suspensions of $\mathrm{Fe} / \mathrm{Fe}_{2} \mathrm{O}_{3}$ nanoparticles capable of providing both $T_{1}$ - and $T_{2}$-weighted images were synthesized. ${ }^{80}$ Similarly, an iron core (with its subsequent oxidation giving a ferrite shell) with added nickel ions to form nickel ferrite shell nanoparticle has been studied; its surface treated with dopamine-PEG to make it dispersible, and it acts as a dual-mode $T_{1}$ and $T_{2}$ contrast agent. ${ }^{81}$

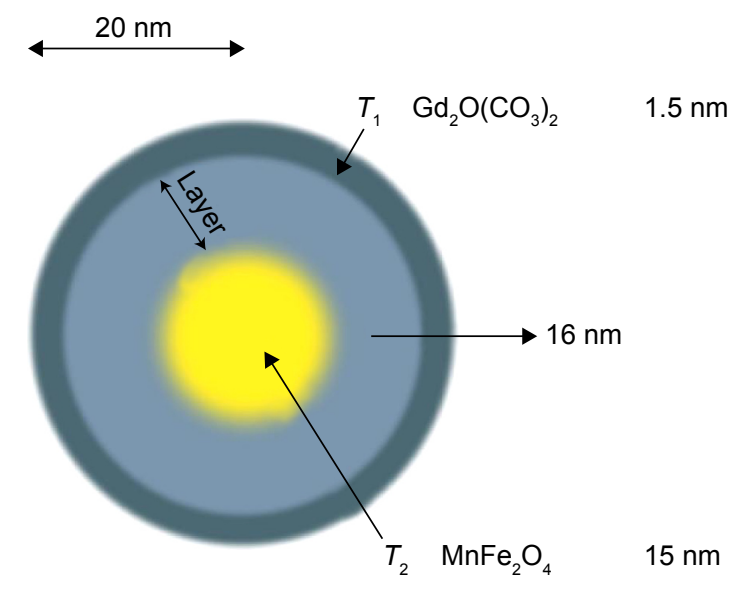

Figure 4 Schematic image of core-shell-type dual-mode nanoparticle contrast agent $\left[\mathrm{MnFe}_{2} \mathrm{O}_{4} @ \mathrm{SiO}_{2} @ \mathrm{Gd}_{2}\left(\mathrm{CO}_{3}\right)_{2}\right]$.

Notes: The $T$, contrast material is positioned on the shell to have direct contact with the water for high $T_{1}$ contrast effects, and the superparamagnetic $T_{2}$ contrast material is located at the core, inducing a long-range magnetic field for the relaxation of water. 
Another approach has been the addition of a Gd chelate to the polymer coating of SPIONs ${ }^{82,83}$ This dual contrast agent efficiently reduces both $T_{1}$ and $T_{2}$ relaxation times and achieved a good contrast in mice for both $T_{1}$ - and $T_{2}$-weighted images. This unique combination allows for the acquisition of both highly detailed $T_{1}$ - and $T_{2}$-weighted images with a single imaging nanoprobe. In addition, such a contrast probe could provide enhanced $T_{1}$-weighted imaging for brain tumors. Common clinical Gd chelates such as Gd-DTPA cannot traverse the blood-brain barrier (BBB) without the use of invasive techniques, ${ }^{84}$ which limits their application in brain tumor imaging. A significant advantage of SPIONs is their relatively large surface area, which allows for the efficient addition of biologically active moieties such as BBBpenetrating peptides for noninvasive brain tumor imaging.

\section{Paramagnetic/superparamagnetic liposomes: versatile MRI probes}

For several years, liposomes have been the center of interest with regard to MRI probes because of their multiple advantages.$^{85}$ One is their capability to encapsulate hydrophilic substances in their aqueous inner core. However, liposomes can, furthermore, encapsulate hydrophobic compounds within a bilayer. Another advantage is their biocompatibility, understood as the quality of having no toxic or injurious effects on biological systems. Both properties enable liposomes to be utilized as carriers, either for therapeutics or diagnostics in vivo. In this way, liposomes have been used to carry either Gd-based contrast agents (paramagnetic liposomes) or Fe-based contrast agents (superparamagnetic liposomes).

As mentioned above in the " $T_{1}$ contrast agents" section, when administered in vivo, Gd chelates rapidly diffuse into tissue and interstitial space and result in decreased lesion/ vessel signal intensity and a concomitantly enhanced signal from surrounding tissues. As a result, contrast between the lesion/vessel and surrounding tissue is reduced, especially in areas where the vasculature is compromised.$^{85}$ Furthermore, due to the latent toxicity of Gd chelates, an efficient renal clearance is highly desirable. ${ }^{19}$ However, for in vitro cell labeling experiments or long-term in vivo cell tracking studies, the clearance of the particles is required to be far lower, which impedes the use of Gd chelates for these purposes, since the rapid reduction in blood concentrations of these agents limits the amount of imaging time available after injection. In this case, active targeting or cell labeling contrast agents must be used. For this, contrast agents must be able to recognize specific molecular sites (eg, cell-specific receptors) at the cellular membrane and to accumulate at those sites. The development of approaches that use probes capable of recognizing and imaging a specific molecular marker of a given pathological process makes diagnosis and therapy much easier. However, the main problem with approaches that use Gd-based contrast agents is the low sensitivity of the resultant MRI, so that to reach $50 \%$ contrast enhancement it is necessary to have a local concentration of contrast agent of the order of $0.5 \mathrm{mM}$. To increase the payload of binding groups delivered to the target site, it is possible to use a single carrier that can bear many ligands to bind to molecular markers. Liposomes and other colloidal structures can be such carriers.

Liposomes can be rendered $T_{1}$ MRI active by the incorporation into the bilayer of Gd conjugated with lipid moieties (for instance, Gd-DTPA-cholesterol) or by encapsulation of Gd within the aqueous space (for instance, in the form of Gd-DTPA) ${ }^{86}$ As a high payload of Gd-containing amphiphilic lipid can be incorporated to the bilayer, the relaxivity per particle increases spectacularly, countering the sensitivity problem..$^{87}$ Nevertheless, the addition of large amounts of Gd alone does not guarantee a low detection limit, since negative effects on the sensitivity may result from a disproportionate increase of $r_{2}$ with respect to $r_{1}$, leading to a reduction of the $r_{1} / r_{2}$ ratio. Moreover, relaxivity rates are a function of the exchange rate of the inner and outer sphere water molecules. As liposome rigidity limits the flux of water between the liposome aqueous cavity and outer bulk water, the physical state of the liposome and its content of cholesterol can modify the overall impact of the contrast agents on local tissue water relaxivity. Moreover, water diffusion at the interface with the phospholipid surface is strongly influenced by the excluded volume provided by the dynamic lipid molecules and head groups. ${ }^{88}$ These facts mean that the lipid composition of liposomes influences the relaxivity produced by the contrast agents encapsulated or incorporated within them.

The encapsulation of a Gd chelate in conventional liposomes results in better circulation properties than those of the free Gd chelate, and encapsulated Gd stays in the circulating blood for longer than free Gd chelate does. However, recognition by serum proteins may compromise the stability of the encapsulated Gd and may thus produce leakage of the $\mathrm{Gd}$ chelate in circulation. The leaked Gd chelate can diffuse into the extravascular space and diminish the quality of the image. Moreover, conventional liposomes are prone to agglomerate when they are obtained at a high lipid concentration, as is usual in these cases. If they were prepared with a low lipid 
concentration, the dose of liposomes required to obtain a significant signal in vivo would involve an excessively high volume of liposomal suspension. The incorporation of PEG into the liposomal membrane by means of a covalent bound creates sterically stabilized liposomes (Stealth ${ }^{\circledR}$ liposomes, SEQUUS Pharmaceuticals, Menlo Park, CA, USA) that have longer blood persistence times. An example of paramagnetic liposomes are PEGylated liposomes encapsulating Gd-DTPA. ${ }^{89,90}$ The relaxation characteristics of Gd PEGylated liposomes differ from those of free Gd-DTPA. As expected, the encapsulation significantly lowers the $r_{1}$ value to $1.04 \mathrm{mM}^{-1} \cdot \mathrm{s}^{-1}$ compared with $4.0 \mathrm{mM}^{-1} \cdot \mathrm{s}^{-1}$ for free Gd-DTPA. The low relaxivity of liposomal Gd is due to the limited access of the Gd atoms to the bulk water molecules, because of the liposomal bilayer, which lowers the rate of water exchange between the bulk and the interior of the liposome. Furthermore, if the lipid contains cholesterol, this reduces the permeability coefficient of the water molecules, leading to a greater reduction in the observed relaxivity. ${ }^{91}$ Size is another factor that strongly affects relaxivity. Small liposomes present higher relaxivities than large liposomes. The difference is due either to the high surface-area-tovolume ratio in small liposomes, which facilitates proton transport across the liposomal bilayer, or to the decreased time of residence of water inside smaller vesicles.

As indicated, the encapsulation of a hydrophilic Gd-based contrast agent in liposomes involves a reduction of the relaxivity. This loss of relaxivity is overcome by an increase in the concentration of Gd in the PEGylated liposomes, which results in a significant shortening of the blood relaxation time $T_{1}$.

Given the relaxivity problems resulting from the encapsulation of $\mathrm{Gd}$ inside liposomes, the incorporation of $\mathrm{Gd}$ chelates into the liposome bilayer is preferable. This approach is expected to enhance the water contact of the Gd chelate, potentially leading to significantly enhanced relaxivity. ${ }^{86}$ To recognize and image a specific molecular marker of a given pathological process or state (MI), such as inflammation, atherosclerosis, angiogenesis, apoptosis, or the presence of tumors, the paramagnetic liposomes are required to target specific molecular markers. Targeted MI of sparsely expressed receptors in tissues, where positive contrast is preferred due to intrinsic $T_{1}$ and $T_{2}$ relaxation times, requires amplification strategies because of the relatively low relaxivities of $T_{1}$-reducing ions, including Gd. Therefore, it is advantageous to use liposomes in which a large payload of paramagnetic lipids can be incorporated into the bilayers, resulting in efficient $T_{1}$ - and $T_{2}$-shortening lipidic nanoparticles. As an example, Gd-DTPA-bis(steraylamide) was incorporated into the lipid bilayer of PEGylated liposomes. Then multiple human recombinant annexin A5 molecules were covalently coupled to introduce specificity for apoptotic cells. The resulting contrast agent increased the relaxation rates of apoptotic cell pellets compared with untreated control cells and apoptotic cells that were treated with nonfunctionalized nanoparticles. ${ }^{92}$

Although recent research has progressed in the preparation and formulation of applications of paramagnetic liposomes for nanoparticle imaging systems, the use of liposomes as contrast agents is mainly based on superparamagnetic liposomes.

Superparamagnetic liposomes are known as magnetoliposomes (MLPs). MLPs were the first multifunctional hybrid liposome/nanoparticle assembly, and they have received considerable attention since their introduction in $1988 .{ }^{93}$ If the superparamagnetic particles are SPIONs, the MLPs can be used as $T_{2}$ contrast agents, whereas if USPIONs are used, the MLPs produce a $T_{1}$ contrast. $^{75,77,94}$ The term MLP denotes several types of phospholipid-iron oxide constructs with totally different properties. The original MLPs consisted of iron oxide cores, upon which a single phospholipid bilayer was adsorbed. The size of such MLPs is approximately $20 \mathrm{~nm}$ (for a review of the original MLPs, see De Cuyper ${ }^{95}$ ). Another kind of MLPs is extruded MLPs, which consist of large unilamellar vesicles (with diameters of the order of a few hundred nanometers) encapsulating several small nanometer-sized water-dispersible iron oxide cores in the aqueous cavity. ${ }^{96,97}$ As an alternative to the extrusion method, encapsulation of magnetic particles can also be achieved by sonication, inverse phase evaporation, or a combination of these techniques. ${ }^{98-100}$ Finally, a third kind of MLPs are formed via the precipitation of iron oxides in the inner space of the vesicles. ${ }^{101}$ This method has many drawbacks (for instance, little control over the size and size distribution, and the need for large amounts of starting ferrous and ferric salts, which greatly affects the $\mathrm{pH}$ and the peroxidation of unsaturated phospholipids). For this reason, "MLPs" usually denotes either original or extruded liposomes.

It has been shown that endosomal localization of different iron oxide particles results in their degradation and reduced MR contrast, the rate of which is governed mainly by the stability of the coating. ${ }^{102}$ The encapsulation of the iron cores in PEGylated liposomes affords, as indicated for paramagnetic liposomes, biological stability that improves the contrast agents. Moreover, unlike in the case of SPIONs, liposomes may have some advantages, especially in the field 
of theranostic agents, eg, nanoscale devices that integrate diagnostic and therapeutic functions. The versatility of lipid types and lipid conjugation permits the combination of the magnetic cores with drugs, fluorescent-lipid conjugates, and ligands to design a single nanosystem that can be detected with multiple imaging techniques. ${ }^{103}$

An additional advantage of MLPs over liposomes or over naked iron oxide nanoparticles is that they can be successfully targeted to body parts of interest, to tumors, for example, and their progression in the body can be followed by MRI. Such targeting can be achieved in two ways: 1) by attaching antibodies or ligands to the vesicle surface that can be selectively recognized by specific receptors present in the cells (biological targeting); ${ }^{104}$ 2) by applying an external magnet near specific body regions where MLPs can then be accumulated (magnetic targeting). ${ }^{105}$ Both approaches allow a reduction in the total number of targeted nanoparticles used compared with untargeted nanoparticles.

MLPs have been used to combine $T_{1}$ and $T_{2}$ MRI contrast agents in a single system to obtain bilabeled contrast agents. ${ }^{106,107} \mathrm{Gd}$ ions were anchored to the surface of MLPs, with up to $500 \mathrm{Gd}$ ions per magnetic vesicle.

Figure 5 is a scheme of a multimodal particle based on a liposomal structure that allows theranostic applications. This liposome contains several individual cores (or a cluster of cores) of SPIONs, and, moreover, it can enclose a drug. The presence of magnetic nanoparticles makes bioimaging possible or the generation of heat in therapeutic hyperthermia, and also magnetic targeting. Optionally, the liposome can also encapsulate a drug. The shell material is responsible for its surface properties, because of the presence of reactive moieties on the surface. In this way, PEG is attached covalently to the surface of phospholipids in order to prevent aggregation and opsonization. The shell can be tuned to provide binding to molecules; as an example, the peptide RGD is bound at the distal end of some PEG chains for the

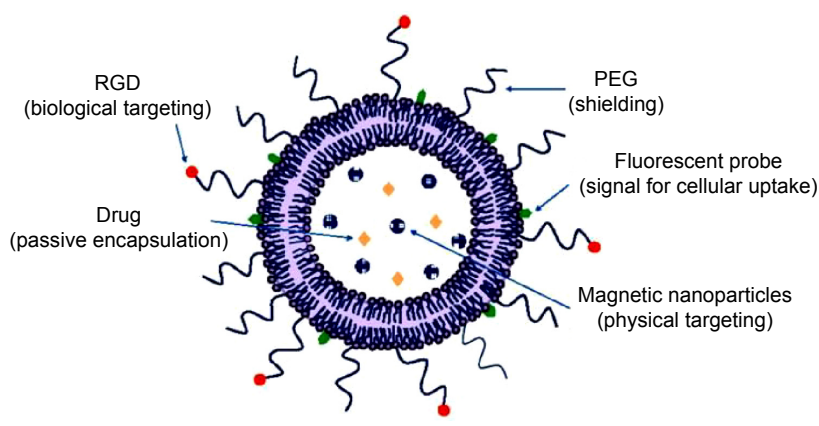

Figure $\mathbf{5}$ Scheme of multifunctional liposome for molecular imaging, drug delivery, and therapy.

Abbreviations: RGD, arginine-glycine-aspartic acid; PEG, poly(ethyelene) glycol. purpose of targeted drug delivery. Such peptides facilitate the interaction of the liposome with integrins: proteins present on the cellular surface that recognize the peptide RGD. This biological targeting promotes the internalization of liposomes into cells. Moreover, the liposomal bilayer can contain a fluorescent probe, which permits its interaction with cells to be visualized by confocal microscopy.

\section{Conclusion}

Over the last 25 years, various nanoparticles and complexes have been studied as MRI contrast agents, and several formulations have been approved for clinical use. These contrast agents are formed either of transition and lanthanide metals or of iron oxide nanoparticles and, more recently, ferrite nanoparticles. The transition or lanthanide metals, whose most significant representative is the ion gadolinium $\left(\mathrm{Gd}^{3+}\right)$, have been extensively used as $T_{1}$ contrast agents since they increase longitudinal relaxation times. A new generation of $T_{1}$ contrast agents is formed by Gd complexes immobilized in various nanostructured materials (nanoporous silicas, dendrimers, perfluorocarbon nanoparticles, and nanotubes). Iron oxide nanoparticles with overall diameters greater than $50 \mathrm{~nm}$ can also be used as MRI contrast agents owing to their capacity to shorten $T_{2}{ }^{*}$ relaxation times in liver, spleen, and bone marrow by selective uptake and accumulation in MPS cells. Iron oxide nanoparticles with diameters $<50 \mathrm{~nm}$ have been used for lymph node imaging; moreover, depending on the size of the iron core and their concentration, these small iron oxide nanoparticles can enhance $T_{1}$ relaxation times. Moreover, iron oxide nanoparticles functionalized with bioactive materials have been used for targeted imaging via the site-specific accumulation.

The presence of dual-mode agents with strong $T_{1}-T_{2}$ contrast effects in a single construct is very challenging, since such dual agents improve the accuracy of biomedical imaging. Moreover, the development of nanomaterials that can filter the MRI artifacts allows the discrimination between signals coming from contrast agents or artifacts.

MLPs are an example of multifunctional platforms for either multimodal imaging or simultaneous imaging and therapy. MLPs can be carefully manipulated in their composition to incorporate cationic lipids, fluorescent-lipid conjugates, targeting ligands, drugs, and PEG, containing all in a single nanosystem.

The most notable limitations associated with the use of such contrast agents are the current detection limits and the lack of tissue specificity. Current detection limits need to be improved for the successful translation of nanoparticles 
to in vivo applications. These limitations have been overcome by recent developments in both MRI acquisition methods and postsynthesis modification of nanoparticles.

\section{Acknowledgment}

The authors are grateful to the Spanish Ministerio de Economía y Competitividad (MINECO) for financial support of the project MAT2012-36270-C04-03.

\section{Author contribution}

All authors contributed toward data analysis, drafting and revising the paper and agree to be accountable for all aspects of the work.

\section{Disclosure}

The authors report no conflicts of interest in this work.

\section{References}

1. Kherlopian AR, Song T, Duan Q, et al. A review of imaging techniques for systems biology. BMC Systems Biol. 2008;2:74.

2. James ML, Gambhir SS. A molecular imaging primer: modalities, imaging agents, and applications. Physiol Rev. 2012;92:897-965.

3. Huang Y, He S, Cao W, Cai K, Liang XJ. Biomedical nanomaterials for imaging-guided cancer therapy. Nanoscale. 2012;4:6135-6149.

4. Jokerst JV, Gambhir SS. Molecular imaging with theranostic nanoparticles. Acc Chem Res. 2011;44:1050-1060.

5. Massoud TF, Gambhir SS. Molecular imaging in living subjects: seeing fundamental biological processes in a new light. Genes Dev. 2003; $17: 545-580$

6. Weissleder R, Ross BD, Rehemtulla A, Gambhir SS. Molecular Imaging: Principles and Practice. Shelton, CT, USA: People's Medical Publishing House; 2010.

7. Rudin M, Weissleder R. Molecular imaging in drug discovery and development. Nature Rev. 2003;2:123-131.

8. Cherry SR. Multimodality in vivo imaging systems: twice the power or double the trouble? Annu Rev Biomed Eng. 2006;8:35-62.

9. Weissleder R, Pitter M. Imaging in the era of molecular oncology. Nature. 2008;452:580-589.

10. Lam T, Pouliot P, Avti PK, et al. Superparamagnetic iron oxide based nanoprobes for imaging and theranostics. Adv Colloid Interface Sci. 2013;199-200:95-113.

11. Opina AC, Ghaghada KB, Zhao P, Kiefer G, Annapragada A, Sherry AD. TmDOTA-tetraglycinate encapsulated liposomes as $\mathrm{pH}$-sensitive LipoCEST agents. PLOS One. 2011;6:e27370.

12. Aime S, Castelli DD, Terreno E. Highly sensitive MRI chemical exchange saturation transfer agents using liposomes. Angew Chem Int Ed. 2005;44:5513-5515.

13. Wu Y, Soesbe TC, Kiefer GE, Zhao P, Sherry D. A responsive europium (III) chelate that provides a direct readout of $\mathrm{pH}$ by MRI. $J$ Am Chem Soc. 2010;132:14002-14003.

14. Hashim Z, Green M, Chung PH, et al. Gd-containing conjugated polymer nanoparticles: bimodal nanoparticles for fluorescence and MRI imaging. Nanoscale. 2014;6:8376-8386.

15. Stephen ZR, Kievit FM, Zhang, M. Magnetic nanoparticles for medical MR imaging. Mater Today. 2011;14:330-338.

16. Mornet S, Vasseur S, Grasset F, et al. Magnetic nanoparticle design for medical diagnosis and therapy. J Mater Chem. 2004;14: 2161-2175.

17. Rümenapp C, Gleich B, Haase A. Magnetic nanoparticles in magnetic resonance imaging and diagnostics. Pharm Res. 2012;29:1165-1179.
18. Laurent S, Vander Elst L, Roch A, et al. Structure, synthesis and characterization of contrast agents for magnetic resonance molecular imaging. In: Carreta P, Lascialfari A, editors. NMR-MRI, SR and Mössbauer Spectroscopies in Molecular Magnets. Milan, Italy: Springer; 2007:71-87.

19. Geraldes CF, Laurent S. Classification and basic properties contrast agents for magnetic resonance imaging. Contrast Media Mol Imaging. 2009;4:1-23.

20. Louie A. Multimodality imaging probes: design and challenges. Chem Rev. 2010;110:3146-3195.

21. Caravan P, Ellison JJ, McMurry TJ, Lauffer RB. Gadolinium (III) chelates as MRI contrast agents: structure, dynamics, and applications. Chem Rev. 1999;99:2293-2352.

22. Wiegers CB, Welch MJ, Sharp TL, et al. Evaluation of two new gadolinium chelates as contrast agents for MRI. Magn Res Imaging. 1992;10:903-911.

23. Lee GH, Chang Y, Kim TJ. Blood-pool and targeting MRI contrast agents: from Gd-chelates to Gd-nanoparticles. Eur J Inorg Chem. 2012; 10:1924-1933.

24. World Health Organization. Pharmaceuticals: Restrictions in Use and Availability. WHO/EMP/QSM/2010.3. Geneva: Switzerland; 2010:14

25. Winter P, Athey P, Kiefer G, et al. Improved paramagnetic chelate for molecular imaging with MRI. J Magn Magn Mater. 2005;293: $540-545$.

26. Kobayshi H, Sato N, Kawamoto S, et al. W. 3D MR angiography of mice using macromolecular MR contrast agents with polyamindoamine dendrimer core with reference to their pharmacokinetic properties. Magn Reson Med. 2001;45:454-460.

27. Frías JC, Williams KJ, Fisher EA, et al. Recombinant HDL-like nanoparticles: a specific contrast agent for MRI of atherosclerosis plaques. J Am Chem Soc. 2004;126:16316-16317.

28. Senpan A, Caruthers SD, Rhee I, et al. Conquering the dark side: colloidal iron oxide nanoparticles. ACS Nano. 2009;3:3917-3926.

29. European Medicines Agency. EMA/486286/2012. London, UK: European Medicines Agency; 2012.

30. Pan D, Caruthers SD, Senpan A, et al. Revisiting and old friend: manganese-based MRI contrast agents. Wiley Interdiscip Rev Nanomed Nanobiotechnol. 2011;3:162-173.

31. Caravan P. Strategies for increasing the sensitivity of gadolinium based MRI contrast agents. Chem Soc Rev. 2006;35:512-523.

32. Park JY, Choi ES, Baek MJ, et al. Water-soluble ultra small paramagnetic or superparamagnetic metal oxide nanoparticles for molecular MR Imaging. Eur J Inorg Chem. 2009;9:2477-2481.

33. Krishnan K. Biomedical nanomagnetics: a spin through possibilities in imaging, diagnostics, and therapy. IEEE Trans Magn. 2010; 46:2523-2558.

34. Filippousi M, Angelakeris M, Sikini M, et al. Surfactant effects on the structural and magnetic properties of iron oxide nanoparticles. $J$ Phys Chem C. 2014;118:16209-16217.

35. Shokrollahi H. Structure, synthetic methods, magnetic properties and biomedical applications of ferrofluids. Mater Sci Eng C Mater Biol Appl. 2013;33:2476-2487.

36. Pflipsen C, Forge D, Benali S, et al. Improved stability and relaxivity of a commercial magnetic ferrofluid. J Phys Chem C. 2013; 117:20919-20926.

37. Bulte JW, Kraitchman DL. Iron oxide MR contrast agents for molecular and cellular imaging. NMR Biomed. 2004;17:484-499.

38. Bulte JW. In vivo MRI cell tracking: clinical studies. Am J Roentgenol. 2009;193:314-325.

39. LaConte LE, Nitin N, Zurkiya O, et al. Coating thickness of magnetic iron oxide nanoparticles affects $\mathrm{R}_{2}$ relaxivity. J Magn Reson Imaging. 2007;26:1634-1641

40. Hu F, Joshi HM, Dravid VP, et al. High-performance nanostructured MR contrast probes. Nanoscale. 2010;2:1884-1891.

41. Choi HS, Liu W, Misra P, et al. Renal clearance of nanoparticles. Nat Biotechnol. 2007;25:1165-1170. 
42. Longmire M, Choyke PL, Kobayashi H. Clearance properties of nanosized particles and molecules as imaging agents: considerations and caveats. Nanomedicine. 2008;3:703-717.

43. Maeda $\mathrm{H}, \mathrm{Wu}$ J, Sawa $\mathrm{T}$, et al. Tumor vascular permeability and the EPR effect in macromolecular therapeutics: a review. J Control Release. 2000;65:271-284

44. Hamm B. Iron-oxide-enhanced MR lymphography: just a new toy or a breakthrough? Eur Radiol. 2002;12;957-958.

45. Lind K, Kresse M, Debus NP, Muller RH. A novel formulation for superparamagnetic iron oxide (SPIO) particles enhancing MR lymphography: comparison of physicochemical properties and the in vivo behavior. J Drug Target. 2002;10:221-230.

46. Maes RM, Lewin JS, Duerk JL, et al. A new type of susceptibilityartefact-based magnetic resonance angiography: intra-arterial injection of superparamagnetic iron oxide particles (SPIO) A Resovist in combination with TrueFisp imaging: a feasibility study. Contrast Media Mol Imaging. 2006;1:189-195.

47. Schmitz SA, Albrecht T, Wolf KJ. MR angiography with superparamagnetic iron oxide: feasibility study. Radiology. 1999;213:603-607.

48. Morawski A, Winter P, Crowder K, et al. Targeted nanoparticles for quantitative imaging of sparse molecular epitopes with MRI. Magn Reson Med. 2004;51:480-486.

49. Cai H, An X, Cui J, et al. Facile hydrothermal synthesis and surface functionalization of polyethyleneimine-coated iron oxide nanoparticles for biomedical applications. ACS Appl Mater Interfaces. 2013;5: 1722-1731.

50. Li J, He Y, Sun W, et al. Hyaluronic acid-modified hydrothermally synthesized iron oxide nanoparticles for targeted tumor MR imaging. Biomaterials. 2014;35:3666-3677.

51. Zhang H, Li J, Sun W, et al. Hyaluronic acid-modified magnetic iron oxide nanoparticles for MR imaging of surgically induced endometriosis model in rats. PLOS One. 2014;9:e94718.

52. Gupta AK, Gupta M. Synthesis and surface engineering of iron oxide nanoparticles for biomedical applications. Biomaterials. 2005;26: 3995-4021.

53. Xie J, Chen K, Lee HY, et al. Ultrasmall c(RGDyK)-coated $\mathrm{Fe}_{3} \mathrm{O}_{4}$ nanoparticles and their specific targeting to integrin alpha(v)-beta(3)rich tumor cells. J Am Chem Soc. 2008;126:11458-11459.

54. Desgrosellier JS, Cheresh DA. Integrins in cancer: biological implications and therapeutic opportunities. Nat Rev Cancer. 2010;10:9-22.

55. Bin Na H, Chan Song I, Hyeon T. Inorganic nanoparticles for MRI contrast agents. Adv Mater. 2009;21:2133-2148.

56. Terreno E, Castelli DD, Viale A, et al. Challenges for molecular magnetic resonance imaging. Chem Rev. 2010;110:3019-3042.

57. Yoo D, Lee JH, Shin TH, et al. Theranostic magnetic nanoparticles. Acc Chem Res. 2011;44:863-874.

58. Hadjipanayis CJ, Bonder MJ, Balakrishnan S, et al. Metallic iron nanoparticles for MRI contrast enhancement and local hyperthermia. Small. 2008;4:1925-1929.

59. Yang H, Zhang C, Shi X, et al. Water-soluble superparamagnetic manganese ferrite nanoparticles for magnetic resonance imaging. Biomaterials. 2010;31:3667-3673.

60. Ho D, Sun X, Sun S. Monodisperse magnetic nanoparticles for theranostic applications. Acc Chem Res. 2011;44:875-882.

61. Jang JT, Nah H, Lee JH, et al. Critical enhancements of MRI contrast and hyperthermic effects by dopant-controlled magnetic nanoparticles. Angew Chem Int Ed. 2009;48:1234-1238.

62. Das GK, Johnson NJ, Cramen J, et al. NaDyF4 nanoparticles as $T_{2}$ contrast agents for ultrahigh field magnetic resonance imaging. $J$ Phys Chem Lett. 2012;3:524-529.

63. Bulte JW, Wu C, Brechbiel MW, et al. Dysprosium-DOTA-PANAM dendrimers as macromolecular $\mathrm{T}_{2}$ contrast agents: preparation and relaxometry. Invest Radiol. 1998;33:841-845.

64. Das GK, Zhang Y, Silva LD, et al. Single-phase $\mathrm{Dy}_{2} \mathrm{O}_{3}: \mathrm{Tb}^{3+}$ nanocrystals as dual-modal contrast agent for field high magnetic resonance and optical imaging. Chem Mater. 2011;23:2439-2446.

65. Gueron M. Nuclear relaxation in macromolecules by paramagnetic ions: a novel mechanism. J Magn Reson. 1975;19:58-66.
66. Taboada E, Rodríguez E, Roig A, et al. Relaxometric and magnetic characterization of ultrasmall iron oxide nanoparticles with high magnetization. Evaluation as potential T1 magnetic resonance imaging contrast agents for molecular imaging. Langmuir. 2007;23:4583-4587.

67. Shubayev VI, Pisanic TR II, Jin S. Magnetic nanoparticles for theragnostics. Adv Drug Deliver Rev. 2009;61:467-477.

68. Cunningham $\mathrm{CH}$, Arai T, Yang PC, et al. Positive contrast magnetic resonance imaging of cells labeled with magnetic nanoparticles. Magn Reson Med. 2005;53:999-1005.

69. Huang J, Zhong X, Wang L, et al. Improving the magnetic resonance imaging contrast and detection methods with engineered magnetic nanoparticles. Theranostics. 2012;2:86-102.

70. Stuber M, Gilson WD, Schär M, et al. Positive contrast visualization of iron oxide-labeled stem cells using inversion-recovery with ON-resonant water suppression (IRON). Magn Reson Med. 2007;58:1072-1077.

71. McAteer MA, Sibson NR, von zur Muhlen C, et al. In vivo magnetic resonance imaging of acute brain inflammation using microparticles of iron oxide. Nature Med. 2007;10:1253-1258.

72. McAteer MA, Schneider JE, Ali ZA, et al. Magnetic resonance imaging of endothelial adhesion molecules in mouse atherosclerosis using dual targeted microparticles of iron oxide. Arterioscler Thromb Vasc Biol. 2008;28:77-83.

73. Von zur Muhlen, von Elverfeldt D, Moeller JA, et al. Magnetic resonance imaging contrast agent targeted toward activated platelets allows in vivo detection of thrombosis and monitoring of thrombolysis. Circulation. 2008;118:258-267.

74. Corot C, Robert P, Idèe JM, et al. Recent Advances in iron oxide nanocrystal technology for medical imaging. Adv Drug Deliv Rev. 2006;58: 1471-1504.

75. Park JY, Daksha P, Lee GH, et al. Highly dispersible PEG surface modified ultra-small superparamagnetic iron oxide nanoparticles useful for target-specific biomedical applications. Nanotechnology. 2008;19:365603.

76. Di Marco M, Sadun C, Port M, et al. Physicochemical characterization of ultrasmall superparamagnetic iron oxide particles (USPIO) for biomedical application as MRI contrast agents. Int J Nanomedicine. 2007;2:609-622.

77. Gossuin Y, Gillis A, Hocq A, et al. Magnetic resonance relaxation properties of superparamagnetic particles. Wiley Interdiscip Rev Nanomed Nanobiotechnol. 2009;1:299-310.

78. Jun YW, Huh YM, Choi JS, et al. Nanoscale size effect of magnetic nanocrystals and their utilization for cancer diagnosis via magnetic resonance imaging. J Am Chem Soc. 2005;127:5732-5733.

79. Choi JS, Lee JH, Shin TH, et al. Self-confirming “AND” logic nanoparticles for fault-free MRI. J Am Chem Soc. 2010;132:11015-11017.

80. Bonmatí MO, Gossuin Y, Morales M, et al. Comparative analysis of the $1 \mathrm{H}$ NMR relaxation enhancement produced by iron oxide and core-shell iron-iron oxide nanoparticles. Magn Reson Imaging. 2007;25:1437-1441.

81. Shultz MD, Calvin S, Fatouros PP, et al. Enhanced ferrite nanoparticles as MRI contrast agents. J Magn Magn Mater. 2007;311:464-468.

82. Bae KY, Kim YB, Lee Y, et al. Bioinspired synthesis and characterization of gadolinium-labeled magnetite nanoparticles for dual contrast $\mathrm{T}_{1}$ - and $\mathrm{T}_{2}$-weighted magnetic resonance imaging. Bioconjugate Chem. 2010;21:505-512.

83. Yang H, Zhuang Y, Sun Y, et al. Targeted dual contrast T1- and T2-weighted magnetic resonance imaging of tumors using multifunctional gadolinium-labeled superparamagnetic iron oxide nanoparticles. Biomaterials. 2011;32:4584-4593.

84. Ding D, Kanaly CW, Cummings TJ, et al. Long-term safety of combined intracerebral delivery of free gadolinium and targeted chemotherapeutic agent PRX321. Neurol Res. 2010;32:810-815.

85. Torchilin VP. Liposomes as delivery agents for medical imaging. Mol Med Today. 1996;2:242-249.

86. Kamaly N, Miller AD. Paramagnetic liposome nanoparticles for cellular and tumour imaging. Int J Mol Sci. 2010;11:1759-1776.

87. Strijkers GJ, Mulder WJ, van Heeswijk RB, et al. Relaxivity of liposomal paramagnetic MRI contrast agents. MAGMA. 2005;18:186-192. 
88. Victor KG, Korb JP, Bryant RG. Translational dynamics of water at the phospholipid interface. J Phys Chem B. 2013;117:12475-12478.

89. Ayyagari LA, Zhang X, Ghaghada KB, Annapragada A, et al. Longcirculating liposomal contrast agents for magnetic resonance imaging. Magn Reson Med. 2006;55:1023-1029.

90. Tilcock C, MacDougall P, Unger E, et al. The effect of lipid composition on the relaxivity of Gd-DTPA entrapped in lipid vesicles of defined size. Biochim Biophys Acta. 1990;1022:181-186.

91. Unger EC, MacDougall P, Cullis P, et al. Liposomal Gd-DTPA: effect of encapsulation on enhancement of hepatoma model by MRI. Magn Reson Imaging. 1989;7:417-423.

92. Van Tilborg GA, Mulder WJ, Deckers N, et al. Annexin A5-functionalized bimodal lipid-based contrast agents for the detection of apoptosis. Bioconjugate Chem. 2006;17:741-749.

93. De Cuyper M, Joniau M. Magnetoliposomes: formation and structural characterization. Eur Biophys J. 1988;15:311-319.

94. Soenen SJ, Hodenius M, De Cuyper M. Magnetoliposomes: versatile innovative nanocolloids for use in biotechnology and biomedicine Nanomedicine (Lond). 2009;4:177-191

95. De Cuyper M. The original magnetoliposomes: from the physicochemical basics to theranostic nanomedicine. In: Callejas-Fernández J, Estelrich J, Quesada-Pérez M, Forcada J, editors. Soft Nanoparticles for Biomedical Applications. Cambridge, UK: Royal Society of Chemistry; 2014.

96. Martina MS, Fortin JP, Menager C, et al. Generation of superparamagnetic liposomes revealed as highly efficient MRI contrast agents for in vivo imaging. J Am Chem Soc. 2005;127:10676-10685.

97. Sabaté R, Barnadas-Rodríguez R, Callejas-Fernández J, et al. Preparation and characterization of extruded magnetoliposomes. Int J Pharm. 2008;347:156-162.
98. Chen Y, Bose A, Bothun GD. Controlled release from bilayerdecorated magnetoliposomes via electromagnetic heating. ACS Nano. 2010;4:3215-3221.

99. García-Jimeno S, Escribano E, Queralt J, et al. Magnetoliposomes prepared by reverse-phase followed by sequential extrusion: characterization and possibilities in the treatment of inflammation. Int $J$ Pharm. 2011;405:181-187.

100. Frascione D, Diwoky C, Almer G, et al. Ultrasmall superparamagnetic iron oxide (USPIO)-based liposomes as magnetic resonance imaging probes. Int J Nanomedicine. 2012;7:2349-2359.

101. Mann S, Hannington JP. Formation of iron oxides in unilamellar vesicles. J Colloid Interface Sci. 1988;122:326-335.

102. Soenen SJ, Himmelreich U, Nuytten N, et al. Intracellular nanoparticle coating stability determines nanoparticle diagnostics efficacy and cell functionality. Small. 2010;6:2136-2145.

103. Fattahi H, Laurent S, Liu F, et al. Magnetoliposomes as multimodal contrast agents for molecular imaging and cancer nanotheragnostics. Nanomedicine. 2011;6:529-544.

104. Casals E, Verdaguer A, Tonda R, et al. Atomic force microscopy of liposomes bearing fibrinogen. Bioconjugate Chem. 2003;14:593-600.

105. García-Jimeno S, Escribano E, Queralt J, et al. External magnetic field-induced selective biodistribution of magnetoliposomes in mice. Nanoscale Res Lett. 2012;7:452.

106. De Cuyper M, Soenen SJ, Coenegrachts K, et al. Surface functionalization of magnetoliposomes in view of improving iron oxide-based magnetic resonance imaging contrast agents: anchoring of gadolinium ions to a lipophilic chelate. Anal Biochem. 2007;367:266-273.

107. Soenen SJ, Desender L, De Cuyper M. Complexation of gadolinium (III) ions on top of nanometer-sized magnetoliposomes. Int J Environ Anal Chem. 2007;87:783-796.
International Journal of Nanomedicine

\section{Publish your work in this journal}

The International Journal of Nanomedicine is an international, peerreviewed journal focusing on the application of nanotechnology in diagnostics, therapeutics, and drug delivery systems throughout the biomedical field. This journal is indexed on PubMed Central, MedLine, CAS, SciSearch $®$, Current Contents $\AA /$ Clinical Medicine,

\section{Dovepress}

Journal Citation Reports/Science Edition, EMBase, Scopus and the Elsevier Bibliographic databases. The manuscript management system is completely online and includes a very quick and fair peer-review system, which is all easy to use. Visit http://www.dovepress.com/ testimonials.php to read real quotes from published authors. 